\title{
Spin-orbit-coupling-induced Fulde-Ferrell-Larkin-Ovchinnikov-like Cooper pairing and skyrmion-like polarization textures in optical lattices
}

\author{
M. Iskin \\ Department of Physics, Koç University, Rumelifeneri Yolu, 34450 Sartyer, Istanbul, Turkey
}

(Received 8 April 2013; published 25 July 2013)

\begin{abstract}
We study the interplay between the Zeeman field and spin-orbit coupling (SOC) in harmonically trapped Fermi gases loaded into a two-dimensional single-band tight-binding optical lattice. Using the Bogoliubov-de Gennes theory, we find that the Zeeman field combined with a Rashba SOC gives rise to (i) Fulde-Ferrell-like superfluidity and (ii) skyrmionlike polarization textures near the edges of the system. We also discuss the effects of interaction, temperature, SOC anisotropy, and Zeeman field anisotropy on the superfluid ground-state and polarization textures.
\end{abstract}

DOI: 10.1103/PhysRevA.88.013631

PACS number(s): 03.75.Ss, 05.30.Fk, 03.75.Hh

\section{INTRODUCTION}

The possibility of simulating non-Abelian artificial gauge fields with quantum Bose and Fermi gases in atomic systems has become one of the forefront research directions in the atomic and molecular physics community [1-9], primarily due to its direct connection to the topological phases of matter that have extensively been studied in the condensed-matter community in recent years [10-13]. In particular, the exciting possibility of the creation and observation of Majorana bound states in topological insulators, superconductors, and superfluids is at the heart of topological quantum computation [14]. These quasiparticles can be created at the boundaries (edges) of non-Abelian topological phases, and they allow for nonlocal storage of quantum information that is protected from local perturbations by the bulk gap. Motivated by these theoretical proposals, spin-orbit coupled Fermi gases have recently been created and detected near the quantum degeneracy limit by three groups $[3,4,6,7]$. While the Shanxi group in China studied the spin dynamics and momentum distribution asymmetry in the equilibrium state as hallmarks of the spin-orbit coupling [3,6], and the Massachusetts Institute of Technology group used a more direct approach and analyzed the energy-momentum dispersion spin-orbit gap and spin composition of the quantum states [4], the NIST group has very recently identified a Feshbach resonance via its associated atomic loss feature [7]. Thus, assuming that sufficiently low temperatures are experimentally attainable in the near future, the physics of Majorana bound states can be studied in the clean and controllable environment uniquely offered by the atomic systems $[15,16]$.

Following the success of these initial experiments [1-7] (see also the recent reviews [8,9]), there has been growing theoretical interest in studying the one-, two-, few-, and many-body properties of spin-orbit coupled Fermi gases. For instance, the stability and phase diagrams have been studied for finite and thermodynamic systems as functions of the interaction strength, spin-orbit coupling (SOC) strength, population imbalance, Zeeman fields, SOC anisotropy, Zeeman field anisotropy, temperature, etc., in one, two, and three dimensions (see, e.g., [17-25]). There also appeared some recent works on the normal-state properties of repulsive Fermi gases with short-range interactions in the upper branch of the spectrum [26], which share similarities with repulsive electron gases with long-range Coulomb interactions [27]. The amount of knowledge gained in these recent works is overwhelming, and here we briefly quote the most recent ones that are concentrated on the possibility of creation and observation of Fulde-Ferrell-Larkin-Ovchinnikov (FFLO) $[28,29]$ type spatially modulated nonuniform superfluid phases under an in-plane Zeeman field [17-25]. In sharp contrast to the out-of-plane Zeeman field, these works have shown that the nonuniform FFLO-like phases are energetically more favored than the uniform BCS-like phases in the case of an in-plane Zeeman field. It is important to note that the FFLO-type phases in spin-orbit coupled Fermi gases are stabilized mainly by the asymmetry of the Fermi surfaces in momentum space, and this mechanism is in contrast with that of the condensed-matter ones, where they are stabilized by the symmetric Zeeman mismatch in momentum space.

Since all of these results are obtained through ansatz-based non-self-consistent momentum-space calculations [17-25], one of our main objectives here is to investigate the stability of FFLO-like phases by solving the Bogoliubov-de Gennes (BdG) equations in a self-consistent fashion. For this purpose, we study the interplay between the Zeeman field and SOC in two-dimensional Fermi gases [30-32] loaded into a singleband tight-binding optical lattice. Our primary finding is that, while the ground states of spin-orbit coupled systems may have weak Fulde-Ferrell (FF) [28] type nonuniform superfluid characters (i.e., phase modulations) but not a LarkinOvchinnikov (LO) [29] one (i.e., amplitude modulations) under the out-of-plane Zeeman field, the FF character of the superfluids is stronger for the Rashba SOC under an in-plane Zeeman field. The FF-type phase oscillations are most prominent along the direction that is perpendicular to the Zeeman field. Therefore, our self-consistent real-space BdG results support recent findings on the thermodynamic continuum systems that are ansatz-based momentum-space calculations [17-25]. We also comment on the effects of interaction, temperature, SOC anisotropy, and Zeeman field anisotropy on the FFLO-like pairing and ground state of the system, and we note that since the superfluid order parameters modulate only toward the edges of the system, where the densities of fermions are low and the magnitudes of the 
order parameters are small, it may be difficult to detect these modulations in atomic systems at finite temperatures.

Furthermore, our secondary finding is that any nonzero combination of the Zeeman field and Rashba SOC induces not only an easy-axis polarization along the direction of the Zeeman field everywhere in the system but also a spatially modulated (ring-shaped in magnitude) transverse polarization near the edges. This is in sharp contrast with the trapped systems with no SOC (and also with the thermodynamic systems with SOC), where only an easy-axis polarization can be induced beyond a threshold Zeeman field. We show that the induced polarization textures are skyrmionlike [10,33] finite-size effects, broadened by the trapping potential, and that their microscopic origin can be traced back to the counterflow of spontaneous spin currents in the case of Rashba SOC. The skyrmion particles were originally proposed in the late $1950 \mathrm{~s}$ by the nuclear physicist T. Skyrme as a model for baryons [33], and they were first observed in condensed-matter physics with quantum-Hall ferromagnets as a result of the interplay between the Zeeman field and Coulomb interactions [34,35]. Note that similar skyrmionlike spin textures were previously predicted in atomic physics for a rotating spinor Bose-Einstein condensate (BEC) [36,37] and spin-orbit coupled BEC [38-40], where skyrmions are spontaneously produced by SOC in the latter case without rotation. We also argue that the transverse polarization textures may be used to probe and characterize the topological phase transitions and the associated Majorana bound states in finite spin-orbit coupled Fermi gases, and we comment on the effects of interaction, temperature, SOC anisotropy, and Zeeman field anisotropy on the polarization textures.

The rest of this paper is organized as follows. In Sec. II, first we introduce the mean-field Hamiltonian and then derive the self-consistency equations for the superfluid order parameter, total number of fermions, and out-of- and in-plane spin polarizations within the $\mathrm{BdG}$ framework. We numerically solve the resultant equations and discuss the obtained results in Sec. III. Finally, the conclusions of this paper are briefly summarized in Sec. IV.

\section{BOGOLIUBOV-DE GENNES THEORY}

The results mentioned above are obtained within the selfconsistent BdG theory in real space as discussed next. First of all, we describe the spin-orbit coupled Fermi gases loaded into a two-dimensional single-band tight-binding optical lattice by the grand-canonical mean-field Hamiltonian:

$$
\begin{aligned}
H= & \sum_{i}\left(-t \sum_{\widehat{\mathbf{e}}} C_{i+\widehat{\mathbf{e}}}^{\dagger} \phi_{i+\widehat{\mathbf{e}}, i} C_{i}+\Delta_{i} c_{\uparrow i}^{\dagger} c_{\downarrow i}^{\dagger}+\text { H.c. }\right) \\
& -\sum_{i, \sigma}\left[\left(\mu+s_{\sigma} h_{z}-V_{i}\right) c_{\sigma i}^{\dagger} c_{\sigma i}+\left(h_{x}-i s_{\sigma} h_{y}\right) c_{\sigma i}^{\dagger} c_{-\sigma i}\right],
\end{aligned}
$$

where the operator $c_{\sigma i}^{\dagger}\left(c_{\sigma i}\right)$ creates (annihilates) a pseudospin $\sigma=\{\uparrow, \downarrow\}$ fermion at lattice site $i$, the spinor $C_{i}^{\dagger}=\left(c_{\uparrow i}^{\dagger}, c_{\downarrow i}^{\dagger}\right)$ denotes the fermion operators collectively, $\widehat{\mathbf{e}}=\{\widehat{\mathbf{x}}, \widehat{\mathbf{y}}\}$ allows only nearest-neighbor hopping with amplitude $t$, and H.c. is the Hermitian conjugate. For a generic non-Abelian gauge field $\mathbf{A}=\left(\alpha \sigma_{y},-\beta \sigma_{x}\right)$, where $\sigma_{e}$ is the Pauli matrix and $\{\alpha, \beta\} \geqslant 0$ are independent parameters characterizing both the strength and the symmetry of the SOC, the $\uparrow$ and $\downarrow$ fermions gain $\phi_{i+\widehat{\mathbf{x}}, i}=e^{-i \alpha \sigma_{y}}$ phase factors for hopping in the positive $\widehat{\mathbf{x}}$ direction and $\phi_{i+\widehat{\mathbf{y}}, i}=e^{i \beta \sigma_{x}}$ phase factors for hopping in the positive $\widehat{\mathbf{y}}$ direction. In addition, the complex number $\Delta_{i}$ is the local mean-field superfluid order parameter (to be specified below), $\mu$ is the chemical potential, $s_{\uparrow}=-s_{\downarrow}=1$, $\mathbf{h} \equiv\left(h_{x}, h_{y}, h_{z}\right)$ is the Zeeman field, and $V_{i}=V_{0} r_{i}^{2}$ is the harmonic confining potential where the distance $r_{i}$ of site $i$ is measured from the center of the lattice.

Using the Bogoliubov transformation, the mean-field Hamiltonian given in Eq. (1) for a two-dimensional $L \times L$ square lattice can be compactly written as a $4 L^{2} \times 4 L^{2}$ matrix-eigenvalue problem [41]:

$$
\sum_{j}\left(\begin{array}{cccc}
T_{\uparrow \uparrow} & T_{\uparrow \downarrow} & 0 & \Delta \\
T_{\downarrow \uparrow} & T_{\downarrow \downarrow} & -\Delta & 0 \\
0 & -\Delta^{*} & -T_{\uparrow \uparrow}^{*} & -T_{\uparrow \downarrow}^{*} \\
\Delta^{*} & 0 & -T_{\downarrow \uparrow}^{*} & -T_{\downarrow \downarrow}^{*}
\end{array}\right)_{i j}\left(\begin{array}{c}
u_{n j}^{\uparrow} \\
u_{n j}^{\downarrow} \\
v_{n j}^{\uparrow} \\
v_{n j}^{\downarrow}
\end{array}\right)=\varepsilon_{n}\left(\begin{array}{c}
u_{n i}^{\uparrow} \\
u_{n i}^{\downarrow} \\
v_{n i}^{\uparrow} \\
v_{n i}^{\downarrow}
\end{array}\right),
$$

where $u_{n i}^{\sigma}$ and $v_{n i}^{\sigma}$ are the components of the $n$th quasiparticle wave function at site $i$, and $\varepsilon_{n} \geqslant 0$ is the corresponding energy eigenvalue. Here, the offsite hopping and onsite energy terms are compactly written as

$$
\begin{aligned}
T_{\sigma \sigma^{\prime}}^{i j}= & -t_{\sigma \sigma^{\prime}}^{i j}-\left[\left(\mu+s_{\sigma} h_{z}-V_{i}\right) \delta_{s_{\sigma} s_{\sigma^{\prime}}}\right. \\
& \left.+\left(h_{x}-i s_{\sigma} h_{y}\right) \delta_{s_{\sigma},-s_{\sigma^{\prime}}}\right] \delta_{i j},
\end{aligned}
$$

where $\delta_{i j}$ is the Kronecker delta. The nonvanishing nearestneighbor hopping elements are $t_{\sigma \sigma}^{i, i+\widehat{\mathbf{x}}}=t \cos \alpha$ and $t_{\uparrow \downarrow}^{i, i+\widehat{\mathbf{x}}}=$ $-t_{\downarrow \uparrow}^{i, i+\widehat{\mathbf{x}}}=-t \sin \alpha$ for the positive $\widehat{\mathbf{x}}$ direction and $t_{\sigma \sigma}^{i, i+\widehat{\mathbf{y}}}=$ $t \cos \beta$ and $t_{\uparrow \downarrow}^{i, i+\widehat{\mathbf{y}}}=t_{\downarrow \uparrow}^{i, i+\widehat{\mathbf{y}}}=i t \sin \beta$ for the positive $\widehat{\mathbf{y}}$ direction. Note that the hopping in the negative directions are simply the Hermitian conjugates and also that the angles $\alpha$ and $\beta$ determine, respectively, the relative strength between the spinconserving particle hopping and spin-flipping SOC terms in the $\widehat{\mathbf{x}}$ and $\widehat{\mathbf{y}}$ directions.

In this paper, we consider only the onsite interactions for which the off-diagonal couplings are $\Delta_{i j}=\Delta_{i} \delta_{i j}$ diagonal in the site index. Therefore, Eq. (2) needs to be solved simultaneously with $\Delta_{i}=g\left\langle c_{\uparrow i} c_{\downarrow i}\right\rangle$, where $g \geqslant 0$ is the strength of the onsite interaction between $\uparrow$ and $\downarrow$ fermions, and $\langle\cdots\rangle$ is a thermal average. In addition, we use $\mu$ to fix the total number of fermions $N=\sum_{i} n_{i}$, where $0 \leqslant n_{i}=$ $\sum_{\sigma}\left\langle c_{\sigma i}^{\dagger} c_{\sigma i}\right\rangle \leqslant 2$ gives the local fermion filling. Once the self-consistent solutions are obtained for the wave functions and the energy spectrum, it is a straightforward task to calculate any of the desired observables. For instance, we are interested in the local polarization vector $\mathbf{p}_{\mathbf{i}} \equiv\left(p_{i x}, p_{i y}, p_{i z}\right)$, the components of which follow from the expectation values of the Pauli spin matrices, i.e., $p_{i v}=\left\langle C_{i}^{\dagger} \sigma_{\nu} C_{i}\right\rangle$, and are given by $p_{i x}=2 \operatorname{Re}\left\langle c_{\uparrow i}^{\dagger} c_{\downarrow i}\right\rangle, p_{i y}=2 \operatorname{Im}\left\langle c_{\uparrow i}^{\dagger} c_{\downarrow i}\right\rangle$, and $p_{i z}=$ $\sum_{\sigma} s_{\sigma}\left\langle c_{\sigma i}^{\dagger} c_{\sigma i}\right\rangle$. Thus, we need the following averages for our 
purposes:

$$
\begin{gathered}
\left\langle c_{\uparrow i} c_{\downarrow i}\right\rangle=\sum_{n}\left[\left(v_{n i}^{\uparrow}\right)^{*} u_{n i}^{\downarrow} f\left(\varepsilon_{n}\right)+u_{n i}^{\uparrow}\left(v_{n i}^{\downarrow}\right)^{*} f\left(-\varepsilon_{n}\right)\right], \\
\left\langle c_{\uparrow i}^{\dagger} c_{\downarrow i}\right\rangle=\sum_{n}\left[\left(u_{n i}^{\uparrow}\right)^{*} u_{n i}^{\downarrow} f\left(\varepsilon_{n}\right)+v_{n i}^{\uparrow}\left(v_{n i}^{\downarrow}\right)^{*} f\left(-\varepsilon_{n}\right)\right], \\
\left\langle c_{\sigma i}^{\dagger} c_{\sigma i}\right\rangle=\sum_{n}\left[\left|u_{n i}^{\sigma}\right|^{2} f\left(\varepsilon_{n}\right)+\left|v_{n i}^{\sigma}\right|^{2} f\left(-\varepsilon_{n}\right)\right],
\end{gathered}
$$

where $f(x)=1 /\left(e^{x / T}+1\right)$ is the Fermi-Dirac distribution function, $T$ is the temperature, and the Boltzmann constant $k_{B}$ is set to unity. We also define the total polarization components as $P_{v}=\sum_{i} p_{i v} / N$, where $v \equiv\{x, y, z\}$. Equations (2)-(6) correspond to the generalization of the $\mathrm{BdG}$ equations to the case of spin-orbit coupled Fermi gases on optical lattices.

\section{NUMERICAL RESULTS}

Having established the BdG formalism, next we present our numerical solutions for the ground-state phases, which are performed on a $41 a \times 41 a$ square lattice with $N=150$ fermions in total, where $a$ is the lattice spacing. We take $V_{0}=$ $0.01 t$ as the strength of the trapping potential and discuss both the Rashba-type symmetric $(\alpha=\beta)$ and asymmetric $(\alpha \neq \beta)$ SOC fields. Note that the experimentally more relevant equal Rashba-Dresselhaus (ERD) SOC [1-9] can be obtained by setting $\alpha=0$. The effects of higher fermion numbers and finite temperature are also briefly mentioned toward the end of the paper.

Before we discuss the aforementioned FFLO-like pairing and polarization textures, we make three important remarks. First, in the absence of a SOC, i.e., when $\alpha=\beta=0$, we know that a sufficiently strong Zeeman field $\mathbf{h}$ (the threshold of which depends on $g$ ) can polarize the system along the easy-axis $(\widehat{\mathbf{h}})$ direction. Second, in the absence of a Zeeman field, i.e., when $\mathbf{h} \equiv(0,0,0)$, we also know that the system is trivially unpolarized no matter what the SOC is. Third, while any combination of Zeeman field (no matter how weak the field is) and SOC in a thermodynamic system may produce a uniform polarization along the easy-axis direction, it does not induce any polarization in the transverse direction, i.e., perpendicular to $\widehat{\mathbf{h}}$. With these remarks in mind, next we show that any nonzero Zeeman field can induce intricate polarization textures near the edges of finite-size spin-orbit coupled Fermi gases under various circumstances.

\section{A. Out-of-plane Zeeman field}

Let us first consider an out-of-plane Zeeman field $\mathbf{h} \equiv$ $\left(0,0, h_{z} \neq 0\right)$, which is perpendicular to our square lattice. The magnitudes and phases of typical ground-state order parameters are illustrated for the Rashba and ERD-like SOCs in Figs. 1(a) and 1(b), respectively, for $h_{z}=0.5 t$. The phases of the order parameters clearly show the $C_{4}$ and $C_{2}$ symmetries of the Hamiltonian for the Rashba and ERD-like SOCs, respectively. These figures suggest that the ERD-like SOC has a stronger FF-type nonuniform superfluid character where the phase of the order parameter has a much larger spatial modulation. Note that the phases have both angular and radial oscillations toward the edges of the system where the densities of the fermions are low and the magnitudes of the order (a)
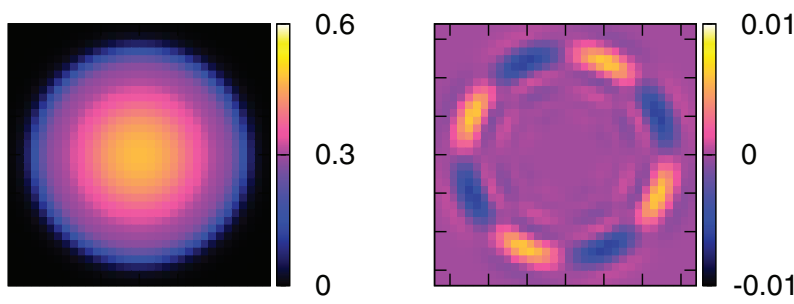

(b)
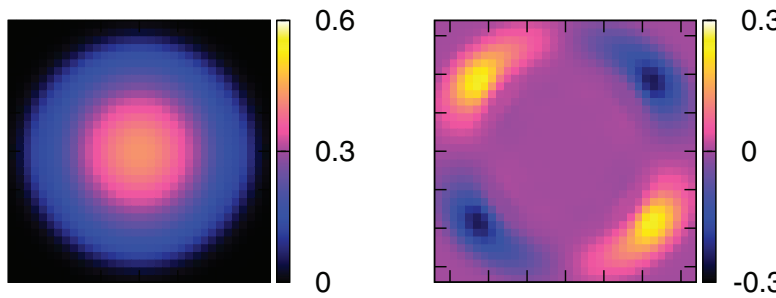

0

FIG. 1. (Color online) The color maps of the amplitudes $\left(\left|\Delta_{i}\right|\right.$, left column) and phases ( $\phi_{i}$, right column) of the order parameters $\Delta_{i}=$ $\left|\Delta_{i}\right| e^{i \phi_{i}}$ for an out-of-plane Zeeman field $\mathbf{h} \equiv(0,0,0.5 t)$ at $g=3 t$ and $T=0$, where $\alpha=\beta=\pi / 4$ in (a), and $\alpha=\pi / 40$ and $\beta=\pi / 4$ in (b).

parameters are small. Therefore, it may be difficult to detect these modulations in atomic systems at finite $T$. However, since the spatial profiles of the magnitudes of the order parameters do not have any zeros (nodes), our results do not feature any LO-type nonuniform superfluidity. We emphasize that these effects become weaker and weaker with decreasing $h_{z}$ in such a way that all of the local phases of the order parameters vanish as $h_{z} \rightarrow 0$.

The corresponding ground-state polarization textures are illustrated in Fig. 2, where we show two-dimensional vector maps of the transverse polarizations $\left(-p_{i x},-p_{i y}\right)$ together with color maps of the easy-axis polarizations $p_{i z}$. Here, we set $g=3 t$ but emphasize that setting it to zero does not lead to any significant change in the results. These figures again clearly show the $C_{4}$ and $C_{2}$ symmetries of the Hamiltonian for the Rashba and ERD-like SOCs, respectively. First of all, the not-so-interesting $p_{i z}$ is finite everywhere in the trap with its maximum value at the center of the system in both figures, and it gradually decreases to zero toward the edges. The case of Rashba SOC is shown in Fig. 2(a), where we find that $p_{i x} \neq 0$ and $p_{i y} \neq 0$ in general, except for the center of the trap. In the ERD-like case when $\alpha \rightarrow 0$ but $\beta=\pi / 4$, we see in Fig. 2(b) that while $p_{i x} \rightarrow 0$ everywhere in the system $p_{i y}$ remains mostly unchanged. Therefore, in the ERD case when $\alpha=0$, a domain wall is formed on the $x$ axis where $p_{i x}=p_{i y}=0$, and such a limiting behavior can be extracted from Fig. 2(b). Similarly, when $\beta=0$ but $\alpha \neq 0$, a domain wall forms on the $y$ axis where $p_{i x}=p_{i y}=0$ (not shown). Thus, we conclude that a nonzero out-of-plane Zeeman field no matter how small it is (not shown) induces spatially modulated transverse polarizations only along those directions where there is SOC. In most cases, the ratio of the transverse to the easy-axis polarizations is around $5-10 \%$. However, we emphasize that while the total easy-axis polarizations are $P_{z} \approx 0.3$ and $\approx 0.36$ in Figs. 2 (a) and 2(b), respectively, the 

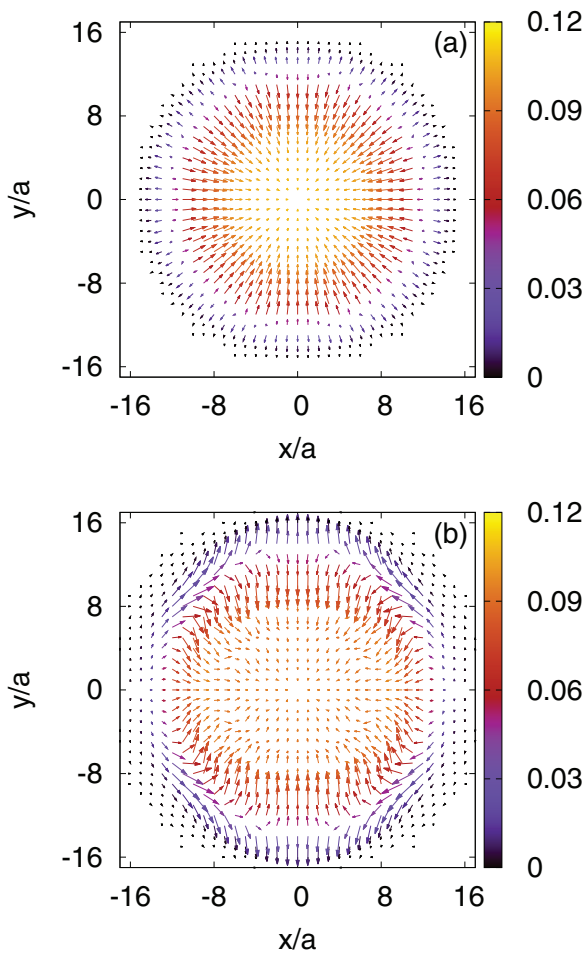

FIG. 2. (Color online) Two-dimensional vector maps of the transverse polarizations $\left(-p_{i x},-p_{i y}\right)$ for an out-of-plane Zeeman field $\mathbf{h} \equiv(0,0,0.5 t)$ at $g=3 t$ and $T=0$. Here, $\alpha=\beta=\pi / 4$ in (a), and $\alpha=\pi / 40$ and $\beta=\pi / 4$ in (b). The largest arrows correspond approximately to 0.01 , and the easy-axis polarizations $p_{i z}$ are illustrated with color maps.

total transverse polarizations vanish, i.e., $P_{x}=0=P_{y}$, as one may expect.

Note that the transverse polarizations change sign in space and that their magnitudes $\sqrt{p_{i x}^{2}+p_{i y}^{2}}$ show ring-shaped structures. In Fig. 2(a), in addition to the broad ring ranging mostly between $7 a \lesssim r_{i} \lesssim 12 a$, there is also a narrow one with a very weak peak around $r_{i} \approx 14 a$. Similarly, in Fig. 2(b), there are two incomplete rings especially along the $y$ axis, and they both have comparable peaks around $r_{i} \approx\{10.5 a, 14.5 a\}$. We also find that increasing the size of the square lattice pushes the ring-shaped structures further away from the center, and reducing the strength of the trapping potential intensifies them around a narrower region near the edges, both eventually leaving no transverse polarization near the center. In the box-potential limit when $V_{0} \rightarrow 0$, we find that while the continuum- and edge-state contributions to the transverse polarizations are competing with each other for low $h_{z}$ values the latter contribution gets stronger with increasing $h_{z}$ and eventually dominates beyond the $h_{z}$ threshold for the creation of zero-energy (Majorana) edge-bound states. These findings suggest that the transverse polarizations observed here are finite-size edge effects, broadened by the trapping potential, and also that the working mechanism is similar to the one that is responsible for the creation of edge-bound Majorana states. We note that similar spin textures are referred to as skyrmions in the contexts of rotating spinor BEC [36,37] and spin-orbit coupled BEC without rotation [38-40]. In particular, we especially note the great similarity between Fig. 2(a) presented here and Fig. 5(a) of Ref. [40].

To understand the microscopic origin of these textures, next we employ the local-density approximation and analyze the single-particle excitation spectrum of the local system. The spectrum of the local Hamiltonian in momentum space involves two quasihole and two quasiparticle branches that are given by

$$
\begin{aligned}
E_{\mathbf{k} i, \pm}^{2}= & \xi_{\mathbf{k} i}^{2}+h_{z}^{2}+\left|s_{\mathbf{k}}\right|^{2}+\left|\Delta_{i}\right|^{2} \\
& \pm 2 \sqrt{h_{z}^{2}\left(\xi_{\mathbf{k}, i}^{2}+\left|\Delta_{i}\right|^{2}\right)+\xi_{\mathbf{k} i}^{2}\left|s_{\mathbf{k}}\right|^{2}}
\end{aligned}
$$

where $\xi_{\mathbf{k} i}=-2 t\left[\cos \alpha \cos \left(k_{x} a\right)+\cos \beta \cos \left(k_{y} a\right)\right]-\mu_{i}$ is the shifted kinetic energy and $\left|s_{\mathbf{k}}\right|^{2}=4 t^{2}\left[\sin ^{2} \alpha \sin ^{2}\left(k_{x} a\right)+\right.$ $\left.\sin ^{2} \beta \sin ^{2}\left(k_{y} a\right)\right]$ is the SOC contribution. Here, the local chemical potential $\mu_{i}=\mu-V_{i}$ includes the trapping potential. We immediately see that the minus branches can become gapless at some k-space points, i.e., $E_{\mathbf{k}_{0} i,-}^{2}=0$, and therefore the locations of zero-energy states are determined by the following conditions: (i) $\left|s_{\mathbf{k}_{0}}\right|=0$ and (ii) $h_{z}=\sqrt{\xi_{\mathbf{k}_{0} i}^{2}+\left|\Delta_{i}\right|^{2}}$. While the Rashba SOC satisfies the former condition at four points $\mathbf{k}_{\mathbf{0}} \equiv\{(0,0) ;(0, \pi) ;(\pi, 0) ;(\pi, \pi)\}$, the ERD SOC satisfies it only at two points $\mathbf{k}_{\mathbf{0}} \equiv\left\{\left(k_{x}, 0\right) ;\left(k_{x}, \pi\right)\right\}$.

It is clear that condition ii is easier to satisfy toward the edges of the system when $\left|\Delta_{i}\right| \rightarrow 0$, and since the transverse polarizations are found to be very similar for $g=3 t$ and $g=0$ we may set $\left|\Delta_{i}\right|=0$ for our purpose. When this is the case, condition ii becomes $\mu_{i}= \pm h_{z} \pm 2 t(\cos \alpha \pm \cos \beta)$ for the Rashba SOC, and $\mu_{i}= \pm h_{z}-2 t\left[\cos \left(k_{x} a\right) \pm \cos \beta\right]$ for the ERD SOC, where all \pm combinations are possible and $\left|\cos \left(k_{x} a\right)\right| \leqslant 1$. For the parameters of Figs. 2(a) and 2(b), where $\mu \approx-1.76 t$, these conditions are satisfied at two distances $r_{i} \approx\{7.5 a, 12.5 a\}$ and $r_{i} \approx\{10.7 a, 14.6 a\}$, respectively, which are very close to our numerical results given above. Thus, we conclude that the microscopic origin of the transverse polarizations can be traced back to the changes in the momentum-space topology of the single-particle excitation spectrum of the local system.

In the case of Rashba SOC, we can also interpret these textures as a direct consequence of counterflow of spontaneously induced spin currents [41]. This is because the Rashba SOC gives rise to an effective momentum-dependent in-plane magnetic field in the direction that is perpendicular to the in-plane momentum. Since the induced spin currents are circulating along the trap edges, i.e., the in-plane momentum is in the azimuthal direction, the induced in-plane spin texture is in the radial direction. The relative contribution between the radially outward and inward helicity bands depends on the local chemical potential $\mu_{i}$, and this competition produces the spatial structure of the spin textures such as the one illustrated in Fig. 2(a). Note that the time-reversal symmetry of the spins must be broken via, e.g., the Zeeman field in order to have a nonzero polarization in any particular direction.

\section{B. In-plane Zeeman field}

Next, we consider an in-plane Zeeman field $\mathbf{h} \equiv\left(0, h_{y} \neq\right.$ 0,0 ), which lies in the $\widehat{\mathbf{y}}$ direction parallel to our square lattice. The magnitudes and phases of typical ground-state order parameters are illustrated for the Rashba and ERD-like SOCs 
(a)

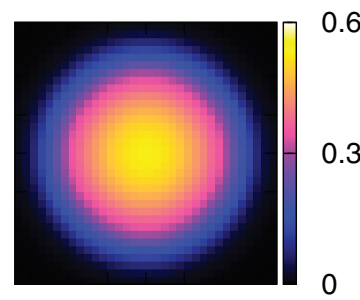

0.6

0.3

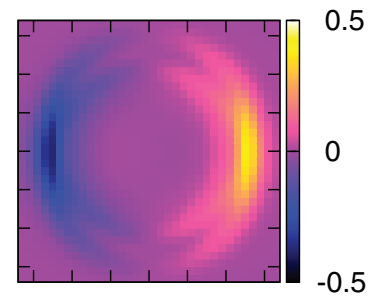

(b)
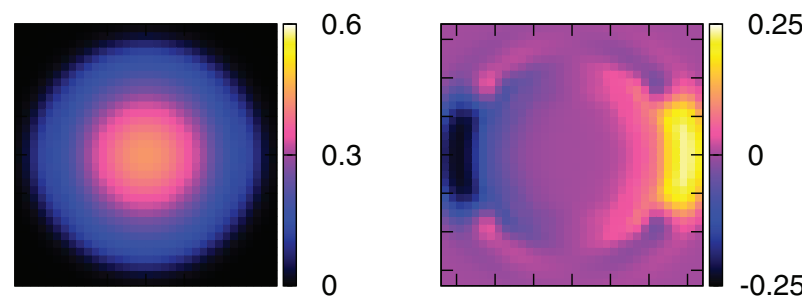

FIG. 3. (Color online) The color maps of the amplitudes $\left(\left|\Delta_{i}\right|\right.$, left column) and phases $\left(\phi_{i}\right.$, right column) of the order parameters $\Delta_{i}=$ $\left|\Delta_{i}\right| e^{i \phi_{i}}$ for an in-plane Zeeman field $\mathbf{h} \equiv(0,0.5 t, 0)$ at $g=3 t$ and $T=0$, where $\alpha=\beta=\pi / 4$ in (a), and $\alpha=\pi / 40$ and $\beta=\pi / 4$ in (b).

in Figs. 3(a) and 3(b), respectively, for $h_{y}=0.5 t$. In sharp contrast to the out-of-plane Zeeman case discussed above, this comparison clearly shows that the Rashba SOC has a much stronger FF-type nonuniform superfluid character in the in-plane Zeeman case, without again featuring any LO-type order parameter node. Note again that the phases have both angular and radial oscillations toward the edges of the system, and the FF-type oscillations are most prominent along the $x$ direction, i.e., perpendicular to the direction of the Zeeman field. We emphasize that these effects become weaker and weaker with decreasing $h_{y}$ in such a way that all of the local phases vanish as $h_{y} \rightarrow 0$.

The corresponding ground-state polarization textures are illustrated in Fig. 4, where we show two-dimensional vector maps of the transverse polarizations $\left(p_{i x}, p_{i z}\right)$ together with color maps of the easy-axis polarizations $p_{i y}$. Here, we set $g=$ $3 t$ but setting $g$ to zero again does not lead to any significant change in the results. First of all, the not-so-interesting $p_{i y}$ is finite everywhere in the trap with its maximum value near the center in both figures, and it gradually decreases to zero toward the edges. The case of Rashba SOC is shown in Fig. 4(a), where we find that $p_{i x} \neq 0$ and $p_{i z} \neq 0$ in general, except for a domain wall on the $x$ axis where $p_{i x}=p_{i z}=0$. In the ERD-like SOC when $\alpha \rightarrow 0$ but $\beta \neq 0$, we see in Fig. 4(b) that while $p_{i x} \rightarrow 0$ everywhere in the system $p_{i z}$ remains mostly unchanged. Therefore, similar to the Rashba case, the ERD case also has a domain wall that is formed on the $x$ axis where $p_{i x}=p_{i z}=0$, and such a limiting behavior can be extracted from Fig. 4(b). On the other hand, when $\beta=0$ but $\alpha \neq 0$, there is not any transverse polarization in the entire system, i.e., $p_{i x}=p_{i z}=0$ for every $i$ (not shown). Thus, we conclude that, when a nonzero in-plane Zeeman field (no matter how small it is) is not perpendicular to the direction of the SOC, a spatially modulated polarization is induced in the transverse direction. In most cases, the ratio of the transverse to the easyaxis polarizations is around $5-15 \%$. However, we emphasize that while the total easy-axis polarizations are $P_{y} \approx 0.37$ and
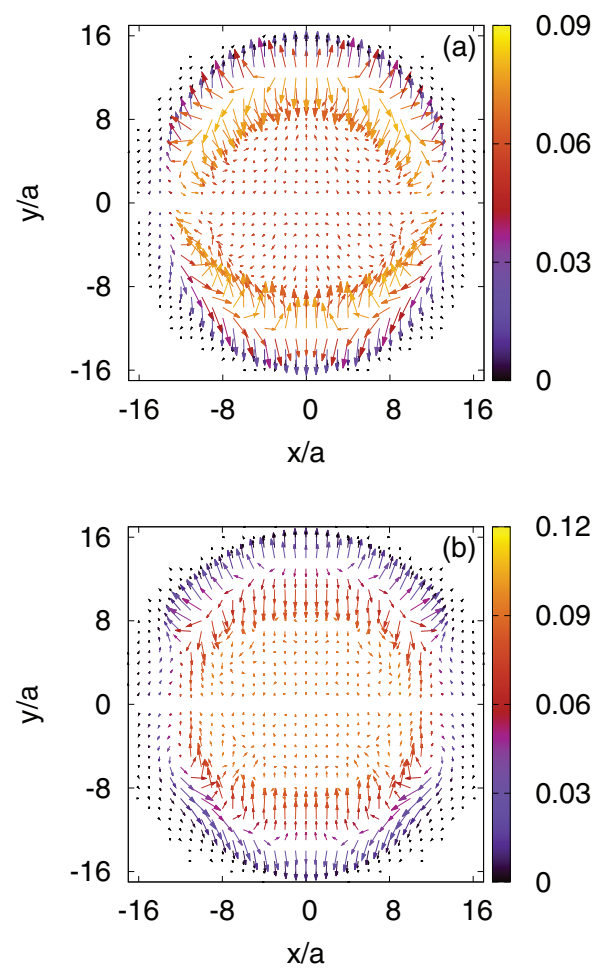

FIG. 4. (Color online) Two-dimensional vector maps of the transverse polarizations $\left(p_{i x}, p_{i z}\right)$ for an in-plane Zeeman field $\mathbf{h} \equiv(0,0.5 t, 0)$ at $g=3 t$. Here, $\alpha=\beta=\pi / 4$ in (a), and $\alpha=\pi / 40$ and $\beta=\pi / 4$ in (b). The largest arrows correspond approximately to 0.014 , and the easy-axis polarizations $p_{i y}$ are illustrated with color maps.

$\approx 0.36$ in Figs. 4(a) and 4(b), respectively, the total transverse polarizations again vanish, i.e., $P_{x}=0=P_{z}$.

Similar to the out-of-plane Zeeman case, we note that the magnitudes of the transverse polarizations $\sqrt{p_{i x}^{2}+p_{i z}^{2}}$ show ring-shaped structures in both Figs. 4(a) and 4(b), where the peaks occur, respectively, at $r_{i} \approx\{10.5 a, 13.5 a\}$ and $\approx\{10.5 \mathrm{a}, 14.5 \mathrm{a}\}$ away from the center of the trap, especially along the $y$ axis. The microscopic origin of these structures can again be traced back to the changes in the momentum-space topology of the single-particle excitation spectrum.

\section{Topological phase transitions}

As elaborated above, although gap closings occur only at a few $\mathbf{k}_{\mathbf{0}}$ points in the lowest quasiparticle and highest quasihole bands, these gapless excitation points are sufficient to induce intricate polarization textures in real space, at and around the boundary between phases with locally different momentum-space topology. We emphasize that since the symmetry of the order parameters of the local phases have the same $s$-wave symmetry across the boundary the transition is topological. Such topological changes are known as Lifshitztype transition in the literature, and they are extensively discussed in the context of nodal, e.g., $p$-wave, superfluids and superconductors [10]. In thermodynamic systems, while the primary signatures of Lifshitz transitions are seen in the momentum distribution and single-particle spectral function, 
some thermodynamic quantities, e.g., atomic compressibility and spin susceptibility, also show anomalies at the transition boundary.

It is also worth noting that both spinless $p_{x} \pm i p_{y}$ (chiral) superfluids [10] and spin-orbit coupled Fermi gases under a Zeeman field $[15,16]$ can host Majorana bound states near the edges of the system. More specifically, these bound states can only exist at the phase boundary between a topologically nontrivial and a trivial phase, the classification of which is based on the value of the topological charges, i.e., Chern numbers [10]. In contrast to the spinless chiral superfluids, our numerical results suggest that the induced transverse polarizations can be used as a probe to characterize the topological phase transitions and the associated Majorana bound states in finite spin-orbit coupled Fermi gases. This is in accordance with a recent work on one-dimensional quantum wires with strong Rashba and Dresselhaus SOC, where it is shown that the Majorana polarization can be used as an order parameter to characterize the topological transition between the trivial system and the system exhibiting Majorana bound modes [42].

We also remark that spin textures may also occur in $p$-wave superfluids (as well as in all other systems with vector order parameters) as topological defects, i.e., a coreless vortex may exist as a spin texture. For instance, such textures were recently observed in superfluid ${ }^{3} \mathrm{He}$ [43], in good agreement with the early predictions $[44,45]$. Furthermore, in the condensedmatter literature, these two-dimensional topological defects were characterized depending on how the local spin changes from the center of the defect to its boundary. Assuming that the local spin $\mathbf{p}_{\mathbf{i}}=p_{i} \widehat{\mathbf{z}}$ is perpendicular to the system at the center of the defect, the topological object is called (a) an Anderson-Toulouse spin texture or a baby skyrmion if the spin continuously rotates through an angle $\pi$ towards the boundary and anti-aligns with respect to the center [45], or (b) a Mermin-Ho spin texture (meron) or a half-skyrmion if the spin continuously rotates through an angle $\pi / 2$ and aligns with the plane of the system [44]. Note that the polarization textures presented in this work do not strictly belong to these classes, and are unique to trapped Fermi gases with SOC.

Having discussed the low-filling Fermi gases at zero temperature, next we briefly comment on the effects of finite $T$ and high fillings. First, although these topological transitions are quantum in their nature, signatures of them can still be observed at finite $T$, where the observables are smeared out due to thermal effects. In particular, we find for the parameters of Figs. 2 and 4 that the maximum magnitudes of the transverse polarizations reduce, respectively, to 50 and $10 \%$ at $T=0.1 t$ and $0.2 t$. Second, due to the particle-hole symmetry of the parent Hamiltonian around half filling, in addition to the ring-shaped transverse polarizations induced near the edges of the system, additional ring-shaped structures are further induced near the center of the trap when the center is close to a band insulator. Therefore, the transverse polarizations show multiple ring-shaped structures in highfilling lattice systems. Having discussed the numerical results, next we conclude the paper with a brief summary of our main findings.

\section{CONCLUSIONS}

In this paper, we studied the interplay between the Zeeman field, SOC, FFLO pairing, and polarization textures in harmonically trapped two-dimensional Fermi gases, loaded into a single-band tight-binding optical lattice. The trapping potential, SOC, and Zeeman field are taken self-consistently into account via the real-space mean-field BdG theory, and two of our main findings can be summarized as follows.

First, we showed that while the ground states of the spin-orbit coupled systems in general have weak FF-type nonuniform superfluid characters but not an LO one under the out-of-plane Zeeman field, the FF character of the superfluids is stronger for the Rashba SOC under an in-plane Zeeman field. The FF-type phase oscillations are also most prominent along the direction that is perpendicular to the Zeeman field. Therefore, our self-consistent results on a finite lattice support recent findings on the thermodynamic continuum systems that are ansatz-based non-self-consistent momentumspace calculations [17-25]. We also discussed the effects of interaction, temperature, SOC anisotropy, and Zeeman field anisotropy on the FFLO-like pairing and ground state of the system, and we noted that since the superfluid order parameters modulate only toward the edges it may be difficult to detect these modulations in atomic systems at finite $T$.

Second, in sharp contrast to the no-SOC case where only an easy-axis polarization is possible beyond a threshold Zeeman field, we showed that any nonzero combination of the Zeeman field and Rashba SOC induces not only an easy-axis polarization everywhere in the system but also a spatially modulated transverse one near the edges. We found that the induced polarization textures are skyrmionlike finite-size effects, which are very similar to the spin textures that were previously predicted for rotating spinor BEC [36,37] and spin-orbit coupled BEC without rotation [38-40]. We also argued that the transverse polarizations can be used to probe and characterize the topological phase transitions and the associated Majorana bound states in finite spin-orbit coupled Fermi gases, and we briefly discussed the possibility of observing these effects in atomic systems.

Finally, we emphasize that while all of these results are obtained using an optical lattice model they are equally applicable to continuum systems in the low-filling limit. We preferred the lattice description mainly because of its easier numerical implementation and versatility, e.g., self-consistent inclusion of the trapping potential, and anisotropic SOC and/or Zeeman field do not require any additional cost in numerics. However, since the lattice model allows particle fillings up to unity, but with a particle-hole symmetry around half filling, it leads to richer finite-size effects compared to the continuum model away from the low-filling limit.

\section{ACKNOWLEDGMENTS}

This work is supported by the Marie Curie International Reintegration Grants Grant No. FP7-PEOPLE-IRG-2010268239, TÜBITAK Career Grant No. 3501-110T839, and TÜBA-GEBIP. The author thanks E. Doko, V. B. Shenoy, and A. L. Subaş1 for discussions. 
[1] Y.-J. Lin, Y.-J. Lin, K. Jiménez-García, and I. B. Spielman, Nature (London) 471, 83 (2011).

[2] J.-Y. Zhang, S.-C. Ji, Z. Chen, L. Zhang, Z.-D. Du, B. Yan, G. S. Pan, B. Zhao, Y. J. Deng, H. Zhai, S. Chen, and J.-W. Pan, Phys. Rev. Lett. 109, 115301 (2012).

[3] P. Wang, Z.-Q. Yu, Z. Fu, J. Miao, L. Huang, S. Chai, H. Zhai, and J. Zhang, Phys. Rev. Lett. 109, 095301 (2012).

[4] L. W. Cheuk, A. T. Sommer, Z. Hadzibabic, T. Yefsah, W. S. Bakr, and M. W. Zwierlein, Phys. Rev. Lett. 109, 095302 (2012).

[5] C. Qu, C. Hamner, M. Gong, C. Zhang, and P. Engels, arXiv:1301.0658 (2013).

[6] Z. Fu, L. Huang, Z. Meng, P. Wang, X.-J. Liu, H. Pu, H. Hu, and J. Zhang, Phys. Rev. A 87, 053619 (2013).

[7] R. A. Williams, M. C. Beeler, L. J. LeBlanc, K. Jiménez-García, and I. B. Spielman, arXiv:1306.1965 (2013).

[8] H. Zhai, Int. J. Mod. Phys. B 26, 1230001 (2012).

[9] V. Galitski and I. B. Spielman, Nature (London) 494, 49 (2013).

[10] G. Volovik, The Universe in a Helium Droplet (Oxford University Press, Oxford, 2003).

[11] M. Z. Hasan and C. L. Kane, Rev. Mod. Phys. 82, 3045 (2010).

[12] X.-L. Qi and S.-C. Zhang, Rev. Mod. Phys. 83, 1057 (2011).

[13] X.-G. Wen, arXiv:1210.1281 (2012).

[14] C. Nayak, S. H. Simon, A. Stern, M. Freedman, and S. das Sarma, Rev. Mod. Phys. 80, 1083 (2008).

[15] M. Iskin, Phys. Rev. A 85, 013622 (2012); 86, 065601 (2012).

[16] X.-J. Liu, L. Jiang, H. Pu, and H. Hu, Phys. Rev. A 85, 021603(R) (2012); X.-J. Liu and H. Hu, ibid. 85, 033622 (2012).

[17] Z. Zheng, M. Gong, X. Zou, C. Zhang, and G.-C. Guo, Phys. Rev. A 87, 031602(R) (2013).

[18] V. B. Shenoy, arXiv:1211.1831 (2012).

[19] L. Dong, L. Jiang, H. Hu, and H. Pu, Phys. Rev. A 87, 043616 (2013).

[20] M. Iskin and A. L. Subaşı, Phys. Rev. A 87, 063627 (2013).

[21] F. Wu, G.-C. Guo, W. Zhang, and W. Yi, Phys. Rev. Lett. 110, 110401 (2013).

[22] X.-J. Liu and H. Hu, arXiv:1302.0553 (2013).

[23] X.-F. Zhou, G.-C. Guo, W. Zhang, and W. Yi, Phys. Rev. A 87, 063606 (2013).
[24] L. Dong, L. Jiang, and H. Pu, New J. Phys. 15, 075014 (2013).

[25] K. Seo, L. Han, and C. A. R. Sá de Melo, arXiv:1301.1353 (2013).

[26] X.-L. Yu, S.-S. Zhang, and W.-M. Liu, Phys. Rev. A 87, 043633 (2013).

[27] A. Ashrafi, E. I. Rashba, and D. L. Maslov, arXiv:1306.1165.

[28] P. Fulde and R. A. Ferrell, Phys. Rev. 135, A550 (1964).

[29] A. I. Larkin and Y. N. Ovchinnikov, Zh. Eksp. Teor. Fiz. 47, 1136 (1964) [Sov. Phys. JETP 20, 762 (1965)].

[30] M. Feld, B. Frohlich, E. Vogt, M. Koschorreck, and M. Köhl, Nature (London) 480, 75 (2011).

[31] E. Vogt, M. Feld, B. Frohlich, D. Pertot, M. Koschorreck, and M. Köhl, Phys. Rev. Lett. 108, 070404 (2012).

[32] A. T. Sommer, L. W. Cheuk, M. J.-H. Ku, W. S. Bakr, and M. W. Zwierlein, Phys. Rev. Lett. 108, 045302 (2012).

[33] T. H. R. Skyrme, Proc. R. Soc. A 260, 127 (1961); Nucl. Phys. 31, 556 (1962)

[34] S. L. Sondhi, A. Karlhede, S. A. Kivelson, and E. H. Rezayi, Phys. Rev. B 47, 16419 (1993).

[35] S. E. Barrett, G. Dabbagh, L. N. Pfeiffer, K. W. West, and R. Tycko, Phys. Rev. Lett. 74, 5112 (1995).

[36] U. A. Khawaja and H. T. C. Stoof, Phys. Rev. A 64, 043612 (2001).

[37] E. J. Mueller, Phys. Rev. A 69, 033606 (2004).

[38] S. Sinha, R. Nath, and L. Santos, Phys. Rev. Lett. 107, 270401 (2011).

[39] C. Wu, I. Mondragon-Shem, and X.-F. Zhou, Chin. Phys. Lett. 28, 097102 (2011).

[40] B. Ramachandhran, B. Opanchuk, X.-J. Liu, H. Pu, P. D. Drummond, and H. Hu, Phys. Rev. A 85, 023606 (2012).

[41] E. Doko, A. L. Subaş1, and M. Iskin, Phys. Rev. A 85, 053634 (2012).

[42] D. Sticlet, C. Bena, and P. Simon, Phys. Rev. Lett. 108, 096802 (2012).

[43] R. Blaauwgeers, V. B. Eltsov, M. Krusius, J. J. Ruohio, R. Schanen, and G. E. Volovik, Nature 404, 471 (2000).

[44] N. D. Mermin and T. L. Ho, Phys. Rev. Lett. 36, 594 (1976).

[45] P. W. Anderson and G. Toulouse, Phys. Rev. Lett. 38, 508 (1977). 